\title{
Tuberculosis versus COVID-19 Mortality: A New Evidence
}

\author{
Hayder Al-Momen ${ }^{1 *}$, Tareef Fadhil Raham ${ }^{2}$, Aqil Mohammad Daher ${ }^{3}$ \\ ${ }^{1}$ Department of Pediatrics, Al-Kindy College of Medicine, University of Baghdad, Baghdad, Iraq; ${ }^{2}$ Department of Pediatrics, \\ Al-Elwiya Pediatric Teaching Hospital, Al-Rusafa Health Directorate, Ministry of Health, Baghdad, Iraq; ${ }^{3}$ Department of \\ Community Medicine, School of Medicine, International Medical University, Bukit Jalil, 57000 KL, Malaysia
}

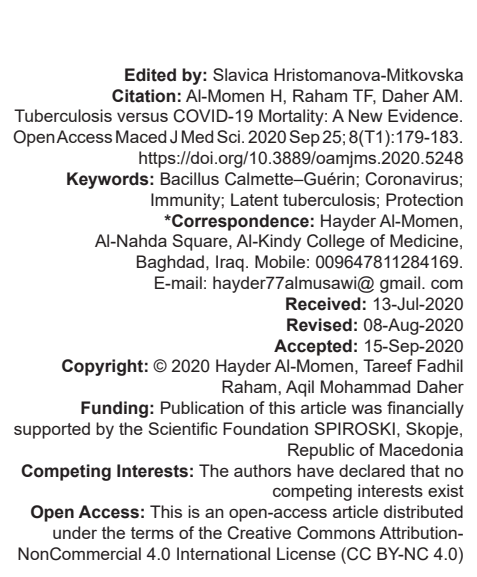

\section{Introduction}

Less than 3 months were needed for the World Health Organization (WHO) to declare coronavirus disease (COVID-19) pandemic on March 11, 2020, since the first cases appeared in Wuhan, China in December, 2019. At that point of the WHO statement, the disease was found in more than 110 countries all over the world. Up to the time of preparing this article, on July 13, 2020; over 12,685,374 people were infected in 216 countries and territories worldwide, with more than 565,000 patients died [1].

The speed of infection and mortality has shown differential models in various countries, United States has the bigger share globally, while Italy and Spain in Europe and Iran and India in Asia did the same within their continents. The number of confirmed cases in a country depends on many factors such as the availability of the diagnostic tests, efficiency of screening programs, and people's cooperation [1], [2].

Mortality rates differ among countries, although the health infrastructure potency is a vital issue, older patients $\geq 65$ years are the main target. Furthermore, comorbidities such as cardiac, renal, liver, and hematological illnesses, on admission D-dimer elevations, and social habits such as smoking were within reported risks. Immunological defects in cellular immunity of $\mathrm{T}$ and $\mathrm{B}$ cells along with later escalation of cytokine cells type 2 productions have the major role toward failure of control over viral replication leading to heavier inflammation and damage, especially in ACE 2 receptors rich organs such as kidneys and lungs with net result of poorer fates [3], [4].

Mycobacterium tuberculosis (Mtb) microorganisms cause tuberculosis (TB) infection which is a common disease worldwide with a clear focus into countries having low and middle-income. One out of four humans on earth is diseased with TB, only $5-15 \%$ of them have symptoms and active illness, so that about $90 \%$ do not show the disease where the host immune response contained Mtb resulting in a condition called latent tuberculosis (LTB). Most of these LTB individuals are missed and living within the community as normal people having the advantage of lifelong immune system activation which starts with innate response aided 
by macrophages and dendritic cells, then T-cellular immune activation will come afterward, especially CD4 and eight, in addition to cytokines distinct role [5], [6].

Trying to take control over the widely-distributed TB disease, a vaccine was developed in the form of live attenuated mycobacterium bovis, called Bacillus Calmette-Guérin (BCG) which has a similar approach of immune stimulation caused by LTB infection including cellular and humeral immune response. This training exercise for the natural immunity either caused by the vaccine or the TB disease itself would offer a protection not limited to Mtb, but extended to other viral infections and respiratory illnesses [7], [8].

Herein, this study; influenced by the distinct protective benefits of the non-specific activation of immune system, we evaluated the association between TB prevalence and mortality rates in different countries affected by COVID-19 pandemic, in the presence or absence of BCG vaccination programs.

\section{Methods}

Available data on public domains for both COVID19 and TB for 191 countries were collected and analyzed. Population size, number of TB cases, and the WHO regions were obtained from the WHO databases along with the classification of economic classes. Data about BCG vaccination policy were obtained from TB world atlas available at (http://www.bcgatlas.org/ index.php. Accessed on July 13, 2020). Data about COVID-19 number of cases and death were obtained from worldometer website available at (https://www. worldometers.info/coronavirus/. Accessed on July 13, 2020). Spearman's correlation test was used for the postulated correlation.

It is noteworthy to mention that we could not control for possible confounding factors including life expectancy, proportion of elderly among population, country preparedness, age, gender, ethnicity, onset of epidemic, migration rate, precision of reporting, and population density.

\section{Results}

We tried not to miss any country in the world registered by the WHO in our analysis. Table 1 shows significant large negative correlation between

Table 1: Worldwide TB cases related to current coronavirus prevalence and mortality

\begin{tabular}{llll}
\hline Overall & Number of countries & Correlation $(\mathrm{R})$ & $\mathrm{p}$ value \\
\hline COVID-19 versus TB cases & 191 & 0.153 & 0.035 \\
COVID-19 death versus TB rate & 191 & -0.539 & $<0.001$ \\
\hline
\end{tabular}

COVID-10 death rate and TB incidence rate per $100 \mathrm{~K}$ population.

Among high income and lower middle-income countries, there is a significant medium negative correlation between COVID-19 death rate and TB incidence rate per $100 \mathrm{~K}$ population, as illustrated in Table 2.

Table 2: Relationship between TB cases and current coronavirus mortality rate according to economic level

\begin{tabular}{llll}
\hline Economic class & Number of countries & Correlation $(R)$ & p value \\
\hline High & 66 & -0.395 & 0.001 \\
Low & 27 & -0.161 & 0.423 \\
Lower medium & 44 & -0.365 & 0.015 \\
Upper medium & 52 & -0.031 & 0.826 \\
\hline
\end{tabular}

There is a significant medium negative correlation between coronavirus pandemic death rate and TB incidence rate per $100 \mathrm{~K}$ population in countries with current BCG vaccination programs. These findings are clear in Table 3.

Table 3: Correlation between rates of TB incidence and COVID-19 mortality with regard to BCG vaccination status

\begin{tabular}{llll}
\hline BCG vaccination status & Number of countries & Correlation $(\mathrm{R})$ & $\mathrm{p}$ value \\
\hline Current & 151 & -0.476 & $<0.001$ \\
No & 19 & -0.299 & 0.213 \\
Previous & 21 & -0.332 & 0.141 \\
\hline
\end{tabular}

\section{Discussion}

Of interest; COVID-19 spread, morbidity and mortality varied widely within various countries, which may be attributed to differences in background culture, health system programs, and migration movements. Scientists all around the globe are racing against time to understand the pathophysiology and behavior of this viral infection aiming to discover a vaccine or proper treatment plan. Theories were proposed along the way, many of them focused on the immune system as the primary defense mechanism against the virus attack.

Accordingly, BCG vaccination was recently put on the table as an immune stimulant to explain the low incidence rates of COVID-19 in countries with routine $B C G$ vaccination practice [8].

This possibility of BCG vaccine could not stand hard in many countries with BCG immunization and high rates of COVID-19 mortality and morbidity providing that the post-BCG vaccine duration of protection has been thought to last only about 10 years [9], although a single report suggested a substantial protection for a longer time [10].

Moreover; some scientists recommended to repeat BCG at any time after at least 3 months' postvaccination because peripheral BCG-induced T-cell memory (effector) population could wane between 3 and 12 months in contrast to central T-cell memory which might persist for decades [11], [12].

All these previously-mentioned BCG related parameters would make it difficult to rely only on 
vaccination data to explain COVID-19 cases as they are usually clustered in older age groups where BCG effects might have been weakened or even disappeared.

Based on the above, we tried something different through focusing on the possible impacts of TB prevalence in affected countries which mirrors the large sector of population carrying latent TB infection when ice burg phenomenon is applied [1], [2].

So that, if one individual has the diagnosis of active TB infection, there has been about nine other people with latent TB diffused within the community and usually unnoticed having the advantage of lifelong immune system stimulation on the contrary of the vaccine where its immune modulation protective effects are vanished with time [9], [10], [11], [12].

We assumed that countries with high TB prevalence have a large population percentage of latent TB individuals whom represent a continuous source of infection and re-infection cycles (mostly asymptomatic) within the community giving it a good chance of ongoing training drills to the immune system making most people resistant to infection complications (including death), a situation which is known as herd immunity [13].

According to our data analysis, we found out a very significant protective (negative) correlation $(-0.539, p<0.001)$ between TB number of cases and COVID-19 mortality rate throughout the world. This crude correlation confirms our above assumption.

Some areas in the world were heavily attacked by COVID-19 leaving more deaths every day. Europe and United States (US) were the major targets of this pandemic regarding deaths per 1 million of population based on worldometer website available at (https:// www.worldometers.info/coronavirus/. Accessed on July 13, 2020). This confusing and unexplained matter urged us to look into each country on earth after dividing them according to the $\mathrm{WHO}$ income classification, regarding TB prevalence and COVID-19 death rates. A medium statistically significant protective correlation appeared in high income countries including Europe and US, and low middle-income countries $(-0.395, p=0.001,-0.365$, $\mathrm{p}=0.015$, respectively). A preceding work suggested higher COVID-19 incidence and death rate in high versus medium economic stage countries based on GDP (gross domestic production) per capita. Most highincome countries have higher means of population age, and dramatic decrease in TB cases in the community with more hygienic environment that necessitating nonroutine use of BCG vaccination [14] which, in turn, might increase COVID-19 burden following our assumption.

\footnotetext{
According to the WHO estimations available at (https://www.who.int/health-topics/ tuberculosis\#tab=tab_1. Accessed on July 13, 2020), half of TB cases in the world were concentrated in eight countries, six of them are low middle-income countries including Bangladesh, India, Pakistan, Indonesia, Nigeria, and the Philippines.
}

At this point, we tried to investigate the already published hypothesis of non-specific BCG vaccination protection effects through immune stimulation; we divided countries on the basis of vaccination status. Current BCG vaccination seemed to have medium protection significant effects to translate the link between high TB prevalence and low COVID-19 mortality.

Most countries with current BCG according to TB world atlas available at (http://www.bcgatlas. org/index.php. Accessed on July 13, 2020) have high TB burden which might be one of the causes that pushed such countries to keep on the continuity of BCG programs. Based on our presumed opinion, it is easy to judge if that low rates of mortality of current coronavirus infection were due to short-term immune activation resulted from BCG vaccine or the natural asymptomatic infection cycles within the community aided by latent TB.

Some reports suggested that countries with up to date continuous BCG immunization programs did better than countries with interrupted or irregular national vaccination policy. Italy and US were good examples of high COVID-19 mortality while they did not experience universal vaccination programs throughout their history [15]. This could be explained by the abovementioned observation of low TB cases in countries without universal BCG vaccination as simply they did not need it.

This was also confirmed by another article which claimed that countries with regular present BCG vaccination had better outcomes than countries with the previous or nil vaccination policy [16].

Flattening of incidence and mortality curves of coronavirus pandemic at each country officially ordering BCG vaccination within a month from the beginning of the wide spread, depending on the distinct correlation between universal BCG policy and COVOD19 prevalence was the findings declared by more researchers [17], [18].

Our data did not show significant effects of the previous BCG vaccination on correlation between TB prevalence and COVID-19 deaths. This is expected according to our hypothesis as it is logical for countries having low TB prevalence to re-evaluate their vaccination strategy and decide to stop BCG, confirming our idea of low TB cases (including latent TB) would leave the community immunity status without activation exercises while early vaccination could not help because of limited protection time. Spain is an example with the previous vaccination policy having one of the highest mortality rates worldwide (deaths per 1 million of population) according to worldometers website available at (https://www.worldometers.info/ coronavirus/. Accessed on July 13, 2020).

This was agreed by other scientists who investigated BCG national coverage during 1 year of age from 1980 to 1985 to find out no significant effects 
on COVID-19 burden [19]. In addition, timing of BCG was not an important risk factor for COVID-19 spread as revealed by some writers [8].

A preceding paper suggested a re-vaccination strategy [20]. Again, this could confirm our idea which says that latent TB could put the community immune system in an alert mode because of high theoretical possibility of continuous infection vicious circles occurred in a natural way in contrast to BCG.

Afresh Japanese paper suggested the protective role of cellular immunity in COVID-19 severity [21]. Although latent TB immune activation is non-specific, cellular immunity is a major participant [7], [8].

It is worthy to say that although concerns were present especially in developed world about latent TB with a potential risk of activation [22], and specific theoretical worries about TB patients when catch COVID-19 infection [23], based on a single retrospective Chinese article that involved only two patients with latent TB but no details of their clinical history [24], there is a clear evidence that latent TB cases represent the immune reactive stage and most of them do not convert into active TB disease even when immune suppressive circumstances have been experienced in line with our alleged consideration [25].

However, many confounding factors could play a role in the mortality or even spread of COVID19 leading to different trajectories in different countries, such as but not limited to fast and effective general protective measures including social crowdedness prevention, biological and genetic factors, presence of comorbidities and chronic illnesses, and environmental pollution, and prevalence of other respiratory viral infections such as different species of influenza virus, and national vaccination program including other vaccines, preparedness of local health system, and pandemic COVID-19 curve stage in each country. Some of these confounders were studied or suggested to be evaluated by the previous workers [8], [14].

We did not involve number of COVID-19 cases in our data analysis due to two main reasons, first because of differences in accuracy levels of COVID19 registry systems in different countries depending on available resources, second cause based on our hypothesis of immune protection offered by latent TB which affects the severity of the disease (COVID-19) rather than the incidence, that is, to say if patients have good immunity, the infection is expected to follow a mild course which may be represented by low mortality.

\section{Conclusion}

Tuberculosis burden has a distinctive crude effect on COVID-19 mortality through our assumption of immune activation theory which is somewhat close to BCG vaccination mechanism but with longer duration resulting in more effective protection against potential lethal nature of current coronavirus pandemic. Confounding factors should be evaluated through controlled clinical trials [26].

\section{Acknowledgments}

The authors would like to show their deep thanks to all medical and editorial staff in Al-Elwiya Teaching Hospital for their kind support.

\section{References}

1. WHO Official Site. Available from: https://www.who.int/ emergencies/diseases/novel-coronavirus-2019. [Last accessed on 2020 Jul 13].

2. Khachfe HH, Chahrour M, Sammouri J, Salhab H, Makki BE, Fares M. An epidemiological study on COVID-19: A rapidly spreading disease. Cureus. 2020;12(3):e7313. https://doi. org/10.7759/cureus. 7313 PMid:32313754

3. Zhou F, Yu T, Du R, Fan G, Liu Y, Liu Z, et al. Clinical course and risk factors for mortality of adult inpatients with COVID-19 in Wuhan, China: A retrospective cohort study. Lancet. 2020;395(10229):1054-62. https://doi.org/10.1016/ s0140-6736(20)30566-3

PMid:32171076

4. Shi Y, Wang Y, Shao C, Huang J, Gan J, Huang X, et al COVID-19 infection: The perspectives on immune responses. Cell Death Differ. 2020;27(5):1451-4. https://doi.org/10.1038/ s41418-020-0530-3

PMid:32205856

5. World Health Organization. Global Tuberculosis Report; 2019. Available from: https://www.apps.who.int/iris/bitstream/han dle/10665/329368/9789241565714-eng.pdf. [Last accessed on 2020 Jul 13].

6. Mack U, Migliori GB, Sester M, Rieder HL, Ehlers $S$, Goletti D, et al. LTBI: Latent tuberculosis infection or lasting immune responses to $M$. tuberculosis? A TBNET consensus statement. Eur Respir J. 2009;33(5):956-73. https://doi. org/10.1183/09031936.00120908

7. De Castro MJ, Pardo-Seco J, Martinón-Torres F. Nonspecific (heterologous) protection of neonatal BCG vaccination against hospitalization due to respiratory infection and sepsis. Clin Infect Dis. 2015;60(11):1611-9. https://doi.org/10.1093/cid/civ144 PMid:25725054

8. Miller A, Reandelar MJ, Fasciglione $\mathrm{K}$, Roumenova $\mathrm{V}, \mathrm{Li} \mathrm{Y}$, Otazu GH. Correlation between Universal BCG Vaccination Policy and Reduced Morbidity and Mortality for COVID-19: An Epidemiological Study. New York: medRxiv; 2020. https://doi. org/10.1101/2020.03.24.20042937

9. Abubakar I, Pimpin L, Ariti C, Beynon R, Mangtani P, Sterne JA, et al. Systematic review and meta-analysis of the current evidence on the duration of protection by bacillus CalmetteGuérin vaccination against tuberculosis. Health Technol 
Assess. 2013;17(37):1-372. https://doi.org/10.3310/hta17370 PMid:24021245

10. Aronson NE, Santosham M, Comstock GW, Howard RS, Moulton LH, Rhoades ER, et al. Long-term efficacy of BCG vaccine in American Indians and Alaska Natives: A 60-year follow-up study. JAMA. 2004;291(17):2086-91. https://doi. org/10.1001/jama.291.17.2086

PMid: 15126436

11. Trnka L, Daňková D, Švandová E. Six years' experience with the discontinuation of BCG vaccination. 2. Cost and benefit of mass BCG vaccination. Tuberc Lung Dis. 1993;74(4):288-92. https://doi.org/10.1016/0962-8479(93)90056-4

PMid:8219182

12. Combadiere B, Boissonnas A, Carcelain G, Lefranc E, Samri A, Bricaire $F$, et al. Distinct time effects of vaccination on longterm proliferative and IFN-gamma-producing $\mathrm{T}$ cell memory to smallpox in humans. J Exp Med. 2004;199(11):1585-93. https:// doi.org/10.1084/jem.20032083 PMid:15184506

13. Anderson RM, May RM. Vaccination and herd immunity to infectious diseases. Nature. 1985;318(6044):323-9. https://doi. org/10.1038/318323a0 PMid:3906406

14. Singh BR, Gandharva R. Are BCG vaccination, population density, median age and poverty important determinants of COVID-19 pandemic spread, morbidity and mortality? Teach Vet Epidemiol. 2020;52(2):1-14.

15. Gursel M, Gursel I. Is global BCG vaccination coverage relevant to the progression of SARS-CoV-2 pandemic? Med Hypotheses. 2020;13(7):109707. https://doi.org/10.1016/j. mehy.2020.109707

16. Sala G, Miyakawa T. Association of BCG Vaccination Policy with Prevalence and Mortality of COVID-19. New York: medRxiv; 2020.

17. Berg MK, Yu Q, Salvador CE, Melani I, Kitayama S. Mandated Bacillus Calmette-Guérin (BCG) Vaccination Predicts Flattened Curves for the Spread of COVID-19. New York: medRxiv; 2020. https://doi.org/10.1126/sciadv.abc1463
18. Dolgikh S. Further Evidence of a Possible Correlation between the Severity of Covid-19 and BCG Immunization. New York: medRxiv; 2020. https://doi.org/10.1101/2020.04.07.20056994

19. Li Y, Zhao S, Zhuang Z, Cao P, Yang L, He D. The correlation between $B C G$ immunization coverage and the severity of COVID-19. SSRN Electron J. 2020;23(8):67-72. https://doi. org/10.2139/ssrn.3568954

20. Dayal D, Gupta S. Connecting BCG Vaccination and COVID-19: Additional Data. New York: medRxiv; 2020. https://doi. org/10.1101/2020.04.07.20053272

21. Yang M, Mao H, Chen L, Zhao L, Lv S, Huang Y, et al. Study on the dynamic change law and correlation between $C T$ imaging manifestations and cellular immunity of 2019 novel coronavirus disease. Jpn J Radiol. 2020;12(1):1-11. https://doi.org/10.1007/ s11604-020-00997-3

PMid:32533391

22. Denholm J, Baker AM, Timlin M. Latent tuberculosis in the general practice context. Aust J Gen Pract. 2020;49(3):107-10. https://doi.org/10.31128/ajgp-10-19-5139 PMid:32113205

23. Maciel EL, Gonçalves Júnior E, Dalcolmo MM. Tuberculosis and coronavirus: What do we know? Epidemiol Serv Saúde. 2020;29(2):e2020128. PMid:32294755

24. Liu Y, Bi L, Chen Y, Wang Y, Fleming J, Yu Y, et al. Active or Latent Tuberculosis Increases Susceptibility to COVID-19 and Disease Severity. New York: medRxiv; 2020. https://doi. org/10.1101/2020.03.10.20033795

25. Behr MA, Edelstein PH, Ramakrishnan L. Is Mycobacterium tuberculosis infection life long? BMJ. 2019;367:I5770. https:// doi.org/10.1136/bmj. 15770

PMid:31649096

26. Bacille Calmette-Guérin Vaccine (BCG) Vaccination and COVID-19. Scientific Brief; 2020. Available from: https://www. apps.who.int/iris/bitstream/handle/10665/331745/WHO-2019nCoV-Sci_Brief-BCGvaccination-2020.1-eng.pdf. [Last accessed on 2020 Jul 13]. https://doi.org/10.15557/pimr.2020.0025 